\title{
Énoncé du Réseau des laboratoires de santé publique du Canada sur les tests de sérologiques au point de soins dans le cadre de la COVID-19
}

\author{
Groupe de travail sur les infections à virus respiratoires ${ }^{1}$
}

\begin{abstract}
Citation proposée : Groupe de travail sur les infections à virus respiratoires. Énoncé du Réseau des laboratoires de santé publique du Canada sur les tests sérologiques au point de soins dans le cadre de la COVID-19. Relevé des maladies transmissibles au Canada 2020;46(5):134-5. https://doi.org/10.14745/ccdr.v46i05a03f

Mots-clés : COVID-19, point de soins, tests sérologiques, Canada, anticorps contre le SRAS-CoV-2
\end{abstract}

\section{Introduction}

Les tests de sérologiques au point de soins pour le coronavirus 2 (SRAS-CoV-2), le virus qui provoque la COVID-19, détectent la réponse de l'anticorps humain à l'infection plutôt que le virus lui-même. La plupart des tests de sérologiques au point de soins sont des tests immunochromatographiques qualitatifs (flux latéral) qui détectent les immunoglobulines $M$ et/ou $G$ dans le sang au moyen d'une piqûre de doigt et peuvent donner des résultats en moins de 30 minutes. Bien que l'on s'intéresse beaucoup à l'adoption de tests de sérologiques au point de soins pour le SRAS-CoV-2, il existe actuellement des limites importantes à cette modalité de test, notamment le manque de compréhension de la réponse immunologique à la COVID-19, les données de validation clinique limitées et la variabilité du rendement entre les différents tests au point de soins.

\section{Position actuelle pour l'utilisation des tests de sérologie au point de soins pour les diagnostics graves}

Les tests sérologiques au point de soins du SRAS-CoV-2 n'ont pas encore été validés pour servir d'outil de diagnostic pour les infections graves et aucun test n'a été approuvé par Santé Canada à ce jour. En général, ces tests de détection des anticorps ne deviennent positifs qu'une semaine ou plus après le début des symptômes et, par conséquent, ne conviennent pas pour le diagnostic d'une infection grave par le SRAS-CoV-2 à ce moment-ci. Nous recommandons que la détection de l'acide nucléique (par exemple, le test en temps réel de réaction en chaîne de la polymérase, le test de RCP) demeure le test de première ligne pour le diagnostic d'une infection grave par le SRAS-CoV-2, comme l'a conseillé l'Organisation mondiale de la Santé (1).

\section{Éléments importants relatifs aux tests de sérologiques au point de soins}

- Les anticorps au SRAS-CoV-2 peuvent se développer de 7 à 12 jours après l'apparition des symptômes; par conséquent,

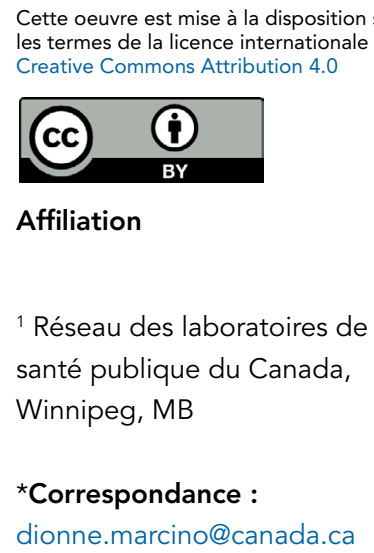

I'utilisation de tests de sérologiques au point de soins au début de l'infection peut donner lieu à de faux résultats négatifs à un moment où les patients sont les plus infectieux (c'est-à-dire, qu'un résultat négatif n'exclut pas l'infection)

- Étant donné que les tests sérologiques au point de soins ne détectent pas le virus, un résultat positif ou négatif ne détermine pas si une personne est infectieuse.

- Les résultats positifs peuvent refléter une infection passée ou présente par le SRAS-CoV-2

- De faux résultats positifs peuvent se produire si ces trousses réagissent à des anticorps provenant d'une exposition récente ou passée à d'autres coronavirus, y compris les coronavirus saisonniers humains (HKU1, NL63, OC43, 229E), le coronavirus respiratoire aigu sévère (SRAS-CoV-1) ou le coronavirus respiratoire du Moyen-Orient (MERS-CoV). D'autres infections, comprenant des maladies non infectieuses (par exemple, les maladies rhumatoïdes à facteur positif), peuvent également produire de faux résultats positifs. Toutes les trousses considérées comme utilisables doivent être soigneusement évaluées pour une telle réactivité croisée avant d'être utilisées en clinique

- De faux résultats négatifs peuvent se produire chez des patients âgés et immunodéprimés

\section{Emplacements où des tests de sérologiques au point de soins pourraient être utilisés}

À l'heure actuelle, I'utilisation de la sérologie dans le diagnostic de l'infection grave par le SRAS-CoV-2 et dans la gestion des patients sera probablement limitée. Cependant, une fois que la dynamique de la réponse sérologique à la COVID-19 sera mieux comprise, la sérologie jouera un rôle important dans la 
réponse en santé publique. L'un des aspects clés de l'utilisation des tests sérologiques consiste à déterminer s'il existe une corrélation possible entre la production d'anticorps et l'immunité protectrice ainsi que la durée de cette protection. La facilité d'utilisation et le temps d'exécution rapide des analyses au point de soins en font une modalité de test idéale dans 1) les régions éloignées ayant un accès limité aux tests centralisés en laboratoire et/ou à une infrastructure locale de laboratoire et

2) des situations qui pourraient bénéficier d'un tri immédiat. En voici des exemples :

- la séroépidémiologie-utilisée pour mieux comprendre la proportion de personnes non diagnostiquées dans la population au fil du temps et pour fournir des données plus précises sur les taux d'attaque et de mortalité

- $\quad$ informer les stratégies de tests diagnostique ciblées (au moyen de tests de RCP), lorsque la priorité serait donnée aux populations ou zones sans preuve d'immunité

- détecter la séroconversion et évaluer l'immunité chez les professionnels de la santé et les autres travailleurs essentiels ou de première ligne

- comme complément au test de RCP pour les tests diagnostiques chez les patients qui ont reçu un résultat négatif au test de la RCP et à la fin de leur maladie pour mettre en œuvre des mesures de contrôle et gérer efficacement les patients

- tester les populations à haut risque exposées au SRAS-CoV-2 pour évaluer leur risque de développer l'infection

- détecter la séroconversion comme substitut pour l'efficacité des mesures de contrôle

- déterminer qui devrait être prioritaire pour une vaccination hâtive lorsque le vaccin est disponible

- $\quad$ soutenir les essais cliniques qui évaluent de nouvelles thérapies, comme l'utilisation d'anticorps neutralisants

\section{Considérations importantes pour la mise en œuvre des tests de sérologiques au point de soins}

- Un test bien validé, qui a été évalué en fonction d'une norme d'excellence en recherche (essais de neutralisation virale ou autre essai sérologique en laboratoire), est nécessaire. Les caractéristiques de rendement (sensibilité, spécificité, valeurs prédictives positives et négatives,

- réaction croisée à d'autres coronavirus) devraient être établies à l'aide de sérums provenant de 1) patients infectés par le SRAS-CoV-2, 2) d'autres virus respiratoires, y compris les coronavirus saisonniers, et 3 ) de contrôles sains

- Une formation adéquate des professionnels de la santé sur I'administration du test et l'interprétation des résultats sera nécessaire

- Le risque d'infection par le SRAS-CoV-2 et les infections transmises par le sang pour l'opérateur doivent être évalués
- Des dispositions doivent être en place pour assurer 1) la saisie de données de dépistage pour les dossiers individuels des patients et à des fins de surveillance et 2) l'obligation de participer à l'évaluation externe de la qualité afin de maintenir des tests de haute qualité

\section{Conclusion}

En se fondant sur l'information actuellement disponible, le Réseau des laboratoires de santé publique du Canada recommande que les tests sérologiques du SRAS-CoV-2 au point de soins ne soient pas utilisés à des fins d'essais cliniques en aucune façon pour le moment. Au fur et à mesure que d'autres renseignements seront disponibles sur le rendement des tests et que les analyses seront validées par rapport aux méthodes sérologiques en fonction d'une norme d'excellence, l'application clinique des tests au point de soins sera réévaluée. Les tests moléculaires, comme le test de RPC en temps réel, demeurent la principale méthode pour confirmer en laboratoire une infection grave par le SRAS-CoV-2 et le diagnostic de la COVID-19.

\section{Déclaration des auteurs}

Le Groupe de travail sur les infections à virus respiratoires du Réseau des laboratoires de santé publique du Canada se consacre à fournir un leadership et des conseils sur les sujets liés aux pathogènes viraux respiratoires, y compris la réponse des laboratoires aux nouveaux virus respiratoires. Le Groupe de travail sur les infections à virus respiratoires est composé de chefs de file de laboratoires de santé publique partout au Canada.

\section{Remerciements}

Le Groupe de travail sur les infections à virus respiratoires tient à remercier les membres du secrétariat du Réseau des laboratoires de santé publique du Canada (RLSPC), S. Radons Arneson et D. Marcino, pour la coordination de la synthèse des documents. Nous tenons également à remercier le Conseil des directeurs de laboratoire du RLSPC pour avoir contribuer à la révision du document.

\section{Référence}

1. World Health Organization. Advice on the use of point-of-care immunodiagnostic tests for COVID-19. WHO, 2020. https://www. who.int/news-room/commentaries/detail/advice-on-the-us e-of-point-of-care-immunodiagnostic-tests-for-covid-19 